\title{
IRÁN 2000: ESTRATEGIAS ELECTORALES Y MECANISMOS DE CONTROL
}

\author{
Dra. D. ${ }^{a}$ María Jesús Merinero Martín \\ Universidad de Extremadura. España
}

Las recientes elecciones legislativas celebradas durante el mes de febrero en Iràn, han desatado no sólo la inmediata euforia interna entre los "jatamistas", sino los elogios de la prensa extranjera sobre la transparencia, pluralidad y civismo demostrado por la sociedad iraní que viene caracterizando a las diversas consultas electorales realizadas desde hace unos años. A la vez que han servido de rcvulsivo para el establishment que ha visto cómo otra de las instituciones democráticas del régimen irani se desvanecía para sus intereses, y se han reforzado las estrategias de poder intentando frenar la capacidad de reformas propuestas por sus adversarios.

No obstante, estas elecciones deben enmarcarse en el importante proceso de democratización y modernización que se está produciendo en Irán en los últimos años con todas las implicaciones que tanto en el ámbito político, social, intelectual c ideológico se están produciendo, en una coyuntura de transformación y recomposición de resultados imprevisibles en la región.

\section{ALGUNAS CONSIDERACIONES SOBRE EL PROCESO POLÍTICO IRANÍ}

El actual proceso se caracteriza por la revolución producida en la vida cotidiana, en las costumbres, la vida social e intelectual, plenamente imbricada en la dialéctica del antagonismo político que ha caracterizado a Irán desde el inicio de la revolución.

Es aún un proceso abierto que políticamente viene marcado por varias cesuras cronológicas -mayo de 1997, con la elección para la Presidencia de la República de Jatamí, líder de la tendencia "reformista", continuada por la victoria de ésta en las elecciones municipales celebradas en la primavera de 1999, las manifestaciones estudiantiles' del 8 al 13 de julio de 1999, que representaron la manifestación pública de tensiones profundamente arraigadas,

1 Un primer acercamiento al tema MERINERO, $\mathrm{M}^{\mathrm{a}} \mathrm{J}$.: “El Irán de las tres revoluciones" en Primer encuentro con Irán. Madrid, Agregación Cultural de la república Islámica de lrán, 2000 (en prensa). 
y las legislativas de febrero de 2000 - sobre el que sólo podemos aventurar hipótesis de futuro, aunque esto no impide que podamos analizar el presente que le caracteriza.

No obstante no es posible comprender en profundidad esta evolución endógena de la sociedad iraní, si no se tienen en cuentas dos factores esenciales: Que la revolución iraní de 1979 es, ante todo, una revolución política. Pues la revolución islámica más allá de ser un movimiento fundamentalista, que en parte lo es, es una revolución politica que ha reformulado la relación religión y política, reforzando este último aspecto ${ }^{2}$. Como tampoco se puede olvidar que la revolución se hizo acorde con la propuesta de síntesis islámico-marxista propuesta por Ali Shariati, uno de los ideólogos más influyentes de la revolución, que facilitó la adhesión a la revolución de una juventud marxista deseosa de una identidad autónoma respecto de Occidente ${ }^{3}$. De tal forma que la revolución asoció inexorablemente la capacidad de lo religioso para consolidar lo político4.

Es necesario, por tanto, rechazar la visión culturalista de la revolución iranis ${ }^{5}$ y dejar de contemplarla como una revancha de la tradición contra la modernización del sha, pues fueron los logros políticos de la propia revolución los que han permitido que los reformistas vayan dominando las instituciones politicas. Por otra parte, si la revolución fue un proceso complejo, en su realización se debe contemplar la existencia de diversos repertorios de actitudes y prácticas, en las que la referencia al islam fue flexible 6 , lo que en su desarrollo posterior ha conducido al debate sobre la democracia y el multipartidismo.

El importante proceso democrático que está llevando a cabo Irán, país clave en el mundo musulmán y en el ámbito del Golfo Pérsico y de Asia Central, está siendo protagonizado y dirigido por una élite politica procedente del mismo sistema -los supervivientes de los enfrentamientos que tuvieron lugar entre 1979 y 1981- lo que confiere al proceso un carácter endógeno. Si la transición consigue felizmente su objetivo, es decir la institucionalización de la soberanía de la ley, y por tanto el establecimiento de un estado de derecho, podrá mantener la legitimidad islámica propia, no sólo de la herencia revolucionaria sino histórica y cultural iraní. A la vez que pondrá de manifiesto la capacidad del islamismo para reformarse y renovarse a partir de sus propios principios o fundamentos -como ya ocurriera en otros momentos del devenir del islam- que permita encontrar una nueva forma de organización socio-política estatal alternativa a los modelos actuales, y mostrar que el islam no es por naturaleza estático y retardatario. Pues la democratización puesta en marcha tiene como finalidad modernizar y adaptar los principios islámicos a la realidad del siglo XXI, lo que debe enmarcarse en la línea de la propuesta hecha por Jomeini con su impulso para

2 Para estos temas, CHEHABI, H.E.: Iranian Politics and religious Modernism. The Liberation Movemem of Iran under the Sahah and Khomeini. Londres, 1990.

3 Para entender esta simbiosis, SHARIATI, A.: Histoire et destinée, textes choisis. Paris, Sindband, 1982.

4 ROY, O.: L'échec de l'islam politique.Paris, Ed du Seuil, 1992.

5 Como ya se encargaron de demostrarlo entre otros DIGARD, J-C.: "Shiisme et État en Iran" en CARRE, O (dir).: L'Islam et l'Etat, Paris, PUF, 1982: pp 65 - 88, RJCHARD, Y.: L 'islam chi'ite. Paris, Fayard, 1991.

6 Para esta variedad de referencias al islam, FISCHER, M.: Iran, from religions dispute to revolution. Cambridge, University Press, 1980. 
"vivir el tiempo presente" o la de otros islamistas como el marroquí Abdalsalam Yasin con la suya de "islamizar la modernidad", que resume esencialmente su doble consideración política: que la falta de democracia es una de las principales causas de la violencia, y que un gobierno democrático debe representar a la sociedad de la que emana, por lo que si una sociedad es musulmana, su gobierno debe tener un carácter islámico. Es decir, acomodar el islam a los cambios y necesidades de las realidades contemporáneas desde un "iytihad, es decir, desde un esfuerzo personal de interpretación, basado en la tradición y la razón?

Realmente, y aunque parezca paradójico, Jatamì no hace más que continuar la herencia de uno de los logros de la revolución que hizo posible un discurso sobre la democracia que es autóctona y no importada, pues provocó una transformación politica, social e intelectual que se revuelve hoy contra su institucionalización anquilosada. De tal forma que la línea reformista procede, no sólo en cuanto se refiere a la génesis de sus élites, sino a la génesis de su ideario, de la propia revolución islámica y se va consolidando teórica y pragmáticamente dentro de ella. Eso explica que los reformistas no cuestionen en su totalidad sus origenes e insistan en que su mensaje renovador, debe interpretarse en gran medida como consecuencia de un proceso de evolución interna y apertura.

Fue la revolución misma la que efectuó una nueva lectura del shiísmo, que consistió en su entrada en el tiempo del mundo, es decir una llamada a la actualización pero sobre todo al abandono de la pasividad shíi en espera de la llegada del imán y una toma de posición activa, lo que significó el inicio de un proceso de secularización del shiismo. De esta forma se entienden las controvertidas declaraciones de destacados reformistas como el ayatolá Montazeri, antiguo delfin de Jomeini, despojado de su sucesión poco antes de la muerte de éste, que propone que el Guía sea elegido por sufragio universal o de Abdullah Nuri afirmando que el Guía es un iraní más que no puede estar por encima de la ley, o de Mohsen Kadivar que pone en entredicho la centralidad del concepto de velayat subrayando el puesto minoritario que ocupa en el edificio conceptual del derecho Shii, y de otros muchos.

Si la experiencia iraní consigue finalizar con éxito, y la próxima oportunidad será la que proporcione las elecciones presidenciales de mayo de 2001, supondrá un revulsivo para los paises árabo-islámicos que les obligará a reflexionar.

\section{LEGITIMIDAD ISLÁMICA, LEGITIMIDAD DEMOCRÁTICA}

La revolución islámica estableció un sistema político basado en la dualidad de legitimidades: La legitimidad islámica, encarnada en la figura del "walayat e-faqih" o gobierno del jurisconsulto, que representa el Guía Supremo (actualmente Jamenei) cuya autoridad se desprende de su conocimiento de los fundamentos islámicos y de su capacidad de interpretarlos correctamente y cuya función consistiría en vigilar la aplicación de los principios islá-

7 LAROUI, A.: Islam et Modernité. Paris, La Découverte, 1986; pp, $135-136$.

8 Para los efectos politicos de la revolución de 1979: KHOROSKHAVAR, F.: Irán de la revolución a la reforma. Barcelona, Bellaterra, 2000. 
micos. Su elección se lleva a cabo mediante una Asamblea de Expertos, elegida democráticamente por el pueblo iraní. La institucionalización del nuevo poder en Irán concedió al Guía supremo unas prerrogativas superiores a las instituciones democráticas pues, al menos en teoría, controla la política exterior, el poder judicial, el Ejército, la policía, los "pasdarán", y los poderosos servicios secretos en el Ministerio de Información. Pero, al margen de la Constitución, controla casi un $80 \%$ de la economía iraní, gracias a un sistema económico estatista, proteccionista, y clientelista, que controla grandes monopolios a través de los beneficios proporcionados a partir de la gestión de las Asociaciones de Mártires y Desheredados, que permiten a ciertos sectores sociales beneficiarse de la revolución?

La legitimidad democrática, representada por la elección mediante sufragio universal, la encarna el Presidente de la República y el Majlis -Parlamento-. Pero la Constitución establece varios centros de poder, representados por los diversos Consejos con gran capacidad de intervención política -Asamblea de Expertos, Consejo de Guardianes de la Constitución, Consejo de Discernimiento del Interés Público, Consejo de Seguridad Nacional.

El Consejo de Guardianes de la Constitución, conocido también como el Consejo de Vigilancia, compuesto por 6 jueces religiosos o laicos designados directamente por el Guia, es el encargado de validar el carácter islámico de las leyes emanadas del Majlis,y supervisa el cumplimiento de la Constitución. Para solucionar las crisis que pueden producirse entre Parlamento y Consejo de Guardianes, que paralizaria la actividad política, se creó el Consejo de Discernimiento del Interés Público, o Defensa de la Razón de Estado, que cuenta desde 1997 con 30 miembros representados por las grandes figuras de la República, es decir, las diversas facciones políticas, lo que obliga a que en su interior exista un consenso mínimo.

Este peculiar sistema constitucional irani que simultanea las instituciones republicanas -Presidente y Parlamento- con el principio shii del "Velayato" o gobierno del jurisconsulto, es una formula que ha servido para dar cabida a las distintas corrientes del régimen, de forma que cada una actuara de contrapeso de las otras. De ahí que la lucha entre facciones se lleve a cabo sobre consideraciones ideológicas y rivalidades intelectuales pero que tenga como uno de los principales objetivos el control de los recursos económicos mediante el ejercicio o el reparto del poder.

La confiscación ${ }^{10}$ de la revolución por el "jomeinismo" la volvió intransigente y corrupta; y politizó el islam hasta tal punto que éste tomó una forma oficial", y propició su reducción a un mecanismo de represión a través del derecho (figh) y del control de las costumbres, y, en consecuencia, fue perdiendo su legitimidad. De hecho, el Guia, designado en 1989, cuya legitimidad fue puesta en entredicho ya desde el principio, ve reducida su legitimidad al arbitraje entre las diferentes tendencias que se dan en el seno del aparato del Estado.

9 Un acercamiento al tema, CARRÉ, O ( dir).: LYslam et l'Étal. Paris, PUF, 1982.

10 BANI SADR, H.: Le complot des ayatollahs. Paris, La Découverte, 1989 y DELANOY, Ch. Y PICHARD, J. P.: Khomeini, la révolution trahie. Paris, Carrére, 1988.

11 MERINERO, M. J.: "Europa y el islam: Relaciones y representaciones" en Cursos de la Cäredra Jean Monnet. Salamanca, Cátedra Jean Monnet, 2000. 
A la par que gracias al juego electoral que permite el sistema, los reformistas han ido fortaleciendo su legitimidad democrática mediante procesos electorales avalados por importantes mayorias.

La elección de Jatami en 1997 se realiza en el seno del aparato del estado legado por la revolución, legitimada con un $70 \%$ de los votos en unas elecciones presidenciales cuya participación fue del $87 \%$ del censo electoral. Para sus partidarios significa el inicio de un capitulo nuevo de gran transcendencia, que se ha dado en llamar la tercera revolución; sus adversarios, que dominan importantes sectores del aparato del estado, ven en ella una puesta en cuestión del velayat bajo disfraz islámico, y por tanto, una puesta en cuestión de la revolución islámica misma.

Lo nuevo en las elecciones de 1997 es que el debate se hace público saliendo de las altas esferas del poder y que una parte de la inteligencia y de la población lo hacen suyo. La habilidad de Jatamí no es tanto proponer una nueva línea como llevar a la escena pública las grandes cuestiones: multipartidismo, estado de derecho, protagonismo de la sociedad civil o diálogo entre las culturas .

La elección de Mohamed Jatamí como presidente de la República, en 1997, puso de manifiesto la existencia de toda una corriente salida de la revolución que aboga por la democratización y modernización del país. Forman el grupo de lo que conocemos en su dimensión política como "reformistas" y en su dimensión ideológica como los "nuevos islamistas".

La democratización del marco institucional desde el interior del sistema, utilizando los mecanismos electorales, que permitan a su vez ejemplificar qué significa un comportamiento acorde con un estado de derecho, es el objetivo político que se ha propuesto Mohamed Jatami, cuya habilidad política ha consistido en reconocer la aspiración de la sociedad a participar activamente en la gestión de los asuntos públicos en el marco de un Estado de derecho. Así se reforzará la legitimidad democrática en detrimento de una muy debilitada legitimidad islámica. Y esta es una de las razones de la transcendencia de las elecciones legislativas de febrero del año 2000.

\section{ELECCIONES LEGISLATIVAS DE FEBRERO DE 2000}

En la estrategia política de los reformistas, de conquista de las instituciones politicas, las elecciones parlamentarias representaban un acontecimiento importante, dado que el Majlis (Parlamento) estaba dominado desde 1992 por los grupos conservadores que impedian poner en práctica muchas de las iniciativas del Gobierno .

Las elecciones legislativas del 18 de febrero de 2000, se han convertido también en un barómetro sobre los treinta meses de presidencia de Jatamí y un pronóstico para las presidenciales del año 2001, por lo que deben enmarcarse en el proceso de democratización iniciado en 1997, pero sin abandonar su transcendencia política para desbloquear las relaciones entre el ejecutivo y el legislativo.

El triunfo de los reformistas en las elecciones legislativas es un importante y transcendente acontecimiento de dimensión nacional e internacional. En el plano nacional, es decir, 
para Irán, este triunfo ha supuesto el triunfo del pueblo irani, de la voluntad popular, de la reafirmación de la legitimidad democrática, puesta de manifiesto en la participación electoral, que ha alcanzado el $83 \%$ del censo electoral, lo que pone de manifiesto el interés de los ciudadanos por participar en la vida política. Pero este entusiasmo por el proceso democrático, no sólo puede medirse por el índice de participación de los votantes, sino también por el de los candidatos. Si el número de escaños parlamentarios que debía cubrirse se había ampliado en 20 para reflejar el incremento de la población, pasando de 270 a 290, la concurrencia de candidatos inscritos para cubrirlos superaba los 6.000 . Y la movilización de las mujeres sigue en aumento, habiéndose inscrito 424 mujeres como candidatos.

La campaña ha sido una campaña limpia en la que los dos candidatos. Alí Reza Jatami y Rafsanyani, han respetado la prohibición de utilizar las descalificaciones personales, a pesar de que su concepción política puede hacer virar el rumbo de la República Islámica. Y porque todos los políticos intentan respetar un código mínimo.

A la vez que, cualitativamente, el modo en que se han celebrado las elecciones ha puesto de manifiesto la autonomía de los ciudadanos para elegir a sus representantes, en un ámbito político plural y diversificado. Ha sido, por tanto, un éxilo esencial para los ciudadanos iranies que, sin duda, están siendo los protagonistas de los grandes cambios.

Un análisis detenido sobre este último proceso electoral ${ }^{12}$ nos permitirá descubrir las estrategias y mecanismos de poder llevadas a cabo por los actores políticos. Pero ¿quiénes son esos actores?

\section{GRUPOS POLÍTICOS}

Aunque los partidos políticos son teóricamente reconocidos por el artículo 26 de la Constitución. De hecho, la escena política no está protagonizada por partidos políticos al estilo occidental, organizados bajo una estricta jerarquización y obediencia de voto en su interior, sino que el régimen se apoya en un sistema de facciones o grupos de naturaleza heteróclita., todos ellos formados por actores de la revolución que salieron victoriosos tras los sobresaltos de 1981 .

Pero lo fundamental de estos grupos políticos heteróclitos no sólo es la diversidad de concepciones sobre la institución estatal, sino la variedad de sus referencias al islam

Estas redes se articulan en torno a asociaciones clericales, a grandes periódicos, a cadenas de negocios y de relaciones personales y familiares Las oposiciones políticas pueden encubrir lazos familiares o personales no menos eficaces. De tal forma que la pertenencia a un grupo no implica unidad de posiciones como lo demuestra ${ }^{13}$ la postura del ayatolá AzariQomi, responsable del grupo de prensa Resalat, vinculado al grupo de la Sociedad del Clero Combatiente, grupo que defiende la herencia del imán Jomeini, que lucha por la defensa de

12 El análisis se efectúa a partir del dossier de prensa, elaborado desde 1997 al 2000 y la consulta a la página web del Frente de Participación.

13 KHOROSKHAVAR, F y ROY, O.: Irán de la revolución a la reforma. Barcelona, Bellaterra, 2000, p. 31. 
la islamización del derecho y de las costumbres, por la preponderancia del clero en el aparato del estado y por la defensa del velayat, y lucha contra el multipartidimo y los liberales. Sin embargo Azari-Qomi manifestó en el otoño de 1997 que Jamenei no debería haber aceptado el cargo de Guía

Si la elección de Jatami polarizó la escena política en dos campos, reformistas y conservadores, no eliminó la complejidad de las lealtades y adhesiones.

Los principales grupos políticos existentes de ámbito nacional son:

Movimiento para la Liberación de Irán (grupo Néhzat-é Azádi ). Organización creada por Mehdi Bazargán, primer ministro tras la caída del Sha. Es un partido laico y liberal.

Partido Nacional del Pueblo de Irán. Partido nacionalista dirigido por el ex ministro de Trabajo del Gobierno provisional Daryoush Foruhar, conocido por sus tomas de posición extremadamente críticas hacia el régimen islámico.

Sociedad del Clero Combatiente. Conjunto clerical conservador cuyo secretario es Mahdavi-Kani, ex fedayin y está apoyado por el grupo de prensa "Resalat". En las elecciones presidenciales de 1997 apoyó la candidatura del clérigo conservador Nátegh-Nurí .

Hasta ahora los conservadores controlaban el aparato del estado y en consecuencia no desean sino mantener la actual situación, Ante los avances de los reformistas que previamente a las elecciones legislativas de 2000, habian conseguido la presidencia de la República y el control de la mayoría, y sobre todo de los más importantes municipios, lo que significa un gran apoyo popular, han procurado una política más moderada, sin atreverse a tomar medidas extremas para detener la liberalización iniciada, por miedo a la reacción popular. No obstante, no han dejado de utilizar sus estrategias electorales ni de poner en marcha los mecanismos de poder que controlan. Dentro de ellos, se pueden distinguir, al menos, dos bloques:

- Línea Muhamad Yazdi, hasta ahora el responsable del poder judicial, que ha controlado estrechamente el poder judicial. Los ayatolás que siguen esta corriente representan la posición más extremista entre los conservadores

- Linea Nateg Nurí, candidato de los conservadores a la presidencia de la República, y hasta ahora presidente del Parlamento, mantienen respecto a los anteriores actitudes más pragmáticas y realistas; la necesidad, cada vez mayor, de llevar a cabo una politica de consenso para no verse definitivamente desplazados, puede que haga variar sino sus planteamientos políticos sí sus actitudes, ante las exigencias aperturistas de la sociedad. Es la línea más implantada en el aparato del estado.

Otra asociación de nombre muy parecido, la de los Clérigos Combatientes, representa la tendencia de la izquierda islámica del clero, pero cuenta también con una base laica con el antiguo primer ministro Musavi. Todos ellos son antiguos activistas de la revolución y partidarios de una estatización de la economía, y su mensaje de justicia social fue muy bien acogida por los más desfavorecidos, pero sus logros distributivos terminaron beneficiando al establishment; sin embargo con el paso del tiempo y la caída del comunismo sus posturas se han ido suavizando y apoyan hoy a Jatamí. Entre ellos hay algunos muy radicales en el plano ideológico, como el ayatolá Sana' y, presidente de la Fundación del 15 de Jordad, que ofre- 
ce una recompensa actualizada a quien mate a Rushdie ${ }^{14}$. Otro ejemplo de las diferencias entre las adscripciones políticas y la toma de posición ideológica.

Servidores de la Reconstrucción, Conocidos a veces en la prensa por los "pragmáticos", es un grupo creado por Rafsanyani para apoyar a los reformistas en las elecciones presidenciales de 1997. Constituido esencialmente por tecnócratas, profesionales y funcionarios que apoyaron las reformas económicas que Rafsanyani había intentado llevar a cabo. Se organizaron en torno a Hussein Karbastchi, alcalde de Teherán. Su defensa de la liberalización económica coincide con el programa de los "reformistas" de Jatamí.

La organización Heyat-é motalefe-yé islami reúne a los medios afines al Bazar. Conservadores y dedicados a los negocios. En las elecciones presidenciales de 1997, su representante apoyó la candidatura conservadora de Nâtég-Nurí. Sin embargo desde esa fecha se ha distanciado de la postura de las Fundaciones, que también están en el mismo campo político conservador, pero a las que acusa de gozar de privilegios exorbitantes en el campo económico. Una parte apoya la política de apertura al exterior de Jatamí.

El Frente 2 de Jordad. Es la agrupación que reúne a los reformistas. No es un grupo homogéneo sino que agrupa desde miembros de la vieja guardia revolucionaria hasta jóvenes universitarios.Su planteamiento genérico es que para salvar la revolución islámica es preciso realizar profundos cambios institucionales para dar preeminencia política a una sociedad pluralista.

Entre la variedad de grupos que le forman, podemos destacar:

- Frente de la Participación Islámica de Irán. Es el grupo liderado por Jatamí . Su espacio político es la izquierda islámica tradicional y entre sus ideólogos destacan Said Hajarián, consejero presidencial, y Abas Abdi, estratega de la ocupación de la embajada de Estados Unidos

- Oficina para la Consolidación de la Unidad. Takim- e Vag-dat-.- Principal organización universitaria de apoyo a Jatami ; junto con la prensa, los intelectuales y las mujeres, forma la punta de lanza del movimiento popular por la reforma política.

- Línea Abdulah Nuri, junto con Mohsen Kadivar es la cabeza visible de quienes, dentro del régimen, se han atrevido a plantear que la religión debe ser compatible con la democracia. Ambos están encarcelados por sus opiniones. Mohsen Kadivar, representa la tendencia intelectual dentro del clero crítica con el velayat y justifica la democracia parlamentaria.

Abdulah Nuri, era el candidato de los reformistas para ocupar el puesto de presidente del Majlis.

Rupturistas. Tal vez el principal representante sea Husein Alí Montazeri, quien fuera delfin de Jomeini hasta que fue apartado en 1988. En arresto domiciliario desde entonces, cuestiona que el Guía de la revolución, sea el representante de Allah y es partidario de que este cargo sea elegido y revocado por instituciones democráticamente elegidas. En la misma línea se encuentran filosofos como Abdulkarim Soroush y otros. 
Existen, además, dos agrupaciones políticas, no legalizadas pero consentidas por el régimen, como el Movimiento para la Liberación de Irán (grupo Néhzat-é Azádi). Organización creada por Mehdi Bazargán, primer ministro tras la caída del Sha. Es un partido laico y liberal. Y el Partido Nacional del Pueblo de Irán. Partido nacionalista dirigido por el ex ministro de Trabajo del Gobierno provisional Daryoush Foruhar, conocido por sus tomas de posición extremadamente críticas hacia el régimen islámico.

Como grupo ilegal existen los Muyahidines del Pueblo, representantes de la extrema izquierda que tras la desaparición de Benisadr hacen un llamamiento a la insurrección y pasan a la oposición clandestina y armada por su enfrentamiento polarizado con el Hezbolá o Partido de la República islámica. Se mantienen en la ilegalidad y tienen su centro en Irak y una oscura financiación.

Una de las novedades en estas elecciones ha sido la creación de dos grandes agrupaciones -aunque han seguido existiendo diversas facciones que han tomado alternativas diferentes, según las circunstancias, como veremos posteriormentc-, que han agrupado a 15 grupos afines a los conservadores y 18 afines a los reformistas.

No obstante hay que tener en cuenta que la iraní es una sociedad compleja en la que hablar de bipolaridad política es una simplificación mutiladora.

Pero la verdadera novedad ha sido la creación del Frente de Participación 2 de Jordad, cuya estrategia "frentista" es decir, la coalición de facciones políticas, organizaciones universitarias y asociaciones cívicas partidarias de un sistema democrático y pluralista, se han unido para derrocar electoralmente a quienes se resisten al cambio.

Esta característica del Frente 2 de Jordad que agrupa a nacionalistas, liberales moderados, liberales radicales, izquierdistas, tecnócratas y "reformistas", si les une el deseo de cambio, no tienen por qué compartir el modo o el ritmo de dicho cambio.

Esta diversidad de puntos de vista se puso de manifiesto cuando Hachemi Rafsanyani aceptó encabezar la lista conservadora en la que se le proponía como presidente del Parlamento, a la vez que se presentaba como uno de los fundadores de los "Sirvientes de la Reconstrucción", facción que apoyaba al Frente de Participación. Es uno de los ejemplos, de la variedad de posiciones en el interior del Frente.

A la vez que si su formación es un acierto electoral, plantea cierto desafio y gran incertidumbre su comportamiento posterior en la Cámara, asentados ya en su mayoria parlamentaria. Sin embargo han dado señales de madurez politica entre tanta variedad de opciones en su interior al retirarse más de 800 candidatos de las listas, respondiendo a los llamamientos hechos desde el frente para no dividir el voto reformista en aquellas circunscripciones donde se presentaban divididos.

A pesar del sistema de facciones, las votaciones se realizan de acuerdo con diversos factores, no únicamente ideológicos: lealtades familiares, clientelistas, económicas, etc. Aunque paulatinamente la politización de la sociedad ha conducido a que las militancias y lealtades tiendan a hacerse en términos políticos, lo que no evita que sigan existiendo las redes personales. 


\section{PROCEDIMIENTOS ELECTORALES}

El Majlis se renueva cada cuatro años. Para estas elecciones existen 290 escaños, habiendo aumentado su número en veinte para hacerle más representativo en relación al crecimiento demográfico producido en el país.

Los candidatos han de inscribirse en el Ministerio del Interior, encargado de la organización de las elecciones. Las elecciones legislativas están previstas como elecciones a dos vueltas. En la primera vuelta los candidatos han de obtener, al menos, el $25 \%$ de los votos para conseguir un escaño. De tal forma que los escaños que no se hayan cubierto por no haber alcanzado los candidatos este porcentaje de votos, se cubrirán a partir de una segunda votación en que el número de candidatos por escaño queda reducido a los dos más votados en la primera vuelta.

Según lo previsto en la Constitución, se reservan cinco escaños para las minorlas religiosas existentes: uno para los zoroástricos, uno para los judios y tres para los cristianos, a quienesno se les exige que alcancen el $25 \%$ de los votos previstos para los restantes candidatos.

Han estado excluidos de su participación electoral el partido demócrata del Kurdistán de Irán (PDK), los partidos autonomistas kurdos y azeríes. Y también el partido monárquico y los muyahidines, partidos ilegales, que se pronunciaron por el boicoteo y la abstención.

Además de la variedad y flexibilidad de las agrupaciones políticas, las elecciones se realizan por el sistema de "listas abiertas", puesto que se vota a la persona y no al partido o agrupación; de tal forma que el votante debe elaborar su propia lista de candidatos. De esta forma la elección se hace más compleja, pero también más abierta y competitiva.

La convergencia de "listas abiertas" y la ausencia de partidos politicos rígidos, permite que un mismo candidato pueda incluirse en varias listas, a veces opuestas o confrontadas. Este ha sido el caso de Rafsanyani, presidente del Parlamento entre 1980 y 1989, y Presidente de la República de 1989 a 1997, dirigente de los Sirvientes de la Reconstrucción, organización creada para apoyar a Jatamí en las presidenciales de 1997, y que apoyaba a los reformistas, que no tuvo inconveniente en encabezar la lista de los conservadores que le ofrecieron el cargo de Presidente del Parlamento.

El sistema de "listas abiertas" ha permitido que los "Servidores de la Reconstrucción" se hayan alineado con Rafsanyani en Teherán y con los reformistas en las provincias.

La financiación de la campaña electoral corre a cargo de los propios candidatos puesto que carecen de financiación pública, por lo que utilizan contribuciones privadas y las donaciones que consiguen de sus seguidores.

Respecto a los electores, tienen derecho al voto todos los iraníes de nacimiento o nacionalidad que hayan cumplido 15 años. En el momento actual ${ }^{15}$, en pleno proceso modernizador y democrático, y con más de un $65 \%$ de población joven menor de 25 años, y el $54 \%$ menor de 18 , el voto juvenil ha mostrado ser más favorable a las posturas más abiertas. 
El Consejo de Guardianes estableció que el recuento se llevaría a cabo manualmente, introduciendo posteriormente los resultados en el ordenador usando el código de cifras atribuido a cada candidato; posteriormente se vuelve a contar un $5 \%$ de las urnas al azar, para asegurarse de que no se han cometido errores. Parece que las autoridades dan por válidos los datos tras ese recuento.

La segunda vuelta ha de llevarse a cabo en un plazo no superior a dos meses. La segunda vuelta electoral, agotó casi los plazos, en una estrategia institucional y se celebró el 5 de mayo de 2000.

\section{ESTRATEGIAS INSTITUCIONALES}

La utilización de las instituciones controladas por el Guia para evitar que los reformistas e independientes dominen los órganos democráticos de la república y puedan llevar a cabo, con menos dificultades, las reformas prometidas, supondrá la puesta en práctica de tres tipos de estrategias: preventivas, coactivas y represivas.

Estrategias preventivas: En el ámbito de las garantías electorales, corresponde al Ministerio del Interior la organización del proceso electoral. No obstante, el Consejo de Guardianes, cuya función es sancionar el carácter islámico de las leyes votadas en el Parlamento, se atribuye, en la práctica, iniciativas más amplias que las previstas en la Constitución; en concreto el veto a la elección de los candidatos al Parlamento y a la presidencia de la República, con el criterio subjetivo de la islamidad del candidato. El modo de nombramiento para el Consejo de Guardianes le convierte en un arma en manos del Guía, ejerciendo así la tutela sobre el conjunto, al permiterse poder eliminar a los adversarios, a la vez que dificulta la entrada de opositores manifiestos en el Parlamento.

De tal forma que si en las elecciones a las presidenciales de 1997, de 238 candidatos, sólo declaró aptos a 4, y los eliminados lo fueron en nombre de su no islamismo o por su incapacidad para tomar las riendas del Estado debido a su inexperiencia. De entre los candidatos para las elecciones presidenciales 9 mujeres inscribieron su candidatura -la más célebre de ellas era A' zam Tâleghàni, hija del ayatolá Tâlégháni, uno de los protagonistas de la revolución islámica muerto el primer año de la revolución que, aunque crítico con la institucionalización del nuevo poder, sigue siendo hasta hoy el símbolo más visible de la unidad revolucionaria- $\mathrm{y}$ todas fueron rechazadas ${ }^{16}$, con la intención de cerrar el paso a Jatami.

En las legislativas de febrero de 2000, el Consejo de Guardianes ha dejado fuera de listas a 760 candidatos, y entre ellos a 30 diputados en ejercicio, generalmente vinculados a la tendencia reformista o a partidos de oposición tolerada.

Muestra de esta extensión injustificada de las competencias del Consejo de Guardianes, que venía descartando a quienes consideraba alejados de la ortodoxia rigorista a partir de criterios subjetivos y sin dar explicaciones, en beneficio de los sectores más

16 YAVARI- D’HELLENCOURT, Nouchine ( dir).: Les Femmes en Iran, pressions sociales et stratégies identitaires. Paris, Edit, du Seuil, 1998. 
inmovilistas, resulta la reciente ley aprobada por el Parlamento, presentada por Jatamí en agosto de 1999, que exige al consejo la justificación por escrito de su veto, y la posibilidad para los candidatos rechazados de recurrir ante sus decisiones.

También por orden del Consejo de Vigilancia, el recuento se hizo de forma manual, conociéndose los resultados oficiales tres días después de finalizadas las votaciones, bajo el pretexto de evitar que los partidarios de uno u otro sector al celebrar su victoria en la calle pudieran provocar alborotos.

Respecto al sistema electoral, los conservadores han pedido en la campaña su reforma, en un doble sentido: elevar la edad para poder votar, situándola en los 17 años, y acabar con un sistema de votación a dos vueltas, eliminando la segunda vuelta, de tal forma que no sólo pretenden establecer un mecanismo que les sea favorable sino reducir, a la vez, el voto juvenil, más favorable a sus adversarios. En este mismo marco preventivo, se sitúa la negativa institucional a limitar la campaña electoral no sólo en el tiempo, apenas una semana para dar a conocer las diferentes propuestas, sino acompañada de la prohibición de carteles públicos. Para ello la excusa fue evitar el posible despilfarro que según algunas opiniones se había producido durante la campaña para las municipales de 1999. No se oculta a nadie que la intención, dado el sistema de listas abiertas, era provocar el desconocimiento de los candidatos del Frente de Participación 2 de Jordad, que en su mayoria, excepto las grandes personalidades políticas, eran jóvenes y sin larga experiencia en la vida pública, beneficiando, en todo caso, a los candidatos más tradicionales, que no necesitaban darse a conocer, bien para evitar el riesgo de aparecer, de nuevo, públicamente.

Otro de los mecanismos utilizados frente al empuje reformista será la "Organización de Propaganda Islámica", principal plataforma de comunicación del sector conservador, alimentada por mucha gente traída de las zonas rurales y los familiares de los héroes y muertos de la guerra, favorecidos por las Fundaciones, movilizados en grandes manifestaciones en moemtos en que los reformistas consiguen algún éxito jurídico, político o informativo.

Las estrategias coactivas destinadas a intimidar o presionar a los adversarios políticos. Para ello pondrán en práctica dos tipos de procedimientos, unos utilizando el control de las instituciones para situar en ellas a sus más duros partidarios en el control de las organizaciones económicas formales o autónomas, que controlan, como fue el nombramiento de Mhamed Foruzandeh como Presidente de la Fundación de los Desheredados- pingüe monopolio de productos y servicios que emplea a cientos de miles de personas y posee un inmenso capital procedente de la fortuna del sha Reza Pahlevi, y que controla sectores claves para potenciales votantes como la vivienda, la agricultura, los subsidios o los transportes, etc. Otros, sobredimensionando sus atribuciones jurídicas para desprestigiar el comportamiento de los reformistas, o para evitar que puedan concurrir al proceso electoral. Su más importante maniobra ha consistido en eliminar a uno de los más populares candidatos reformistas,el ex ministro del Interior, y uno de los candidatos más votados en las elecciones municipales de 1999, Abdullah Nuri, urdiendo una acusación judicial por propaganda "antiislámica" a partir de las publicaciones realizadas en el periódico que dirige "Jordad"17, deján- 
doles sin candidato unánime para la presidencia del Parlamento, a la vez que ofrecían este puesto en su candidatura a Rafsanyani, pensando que podría atraer votos de los más moderados y de los indecisos.

La inclusión de Rafsanyani como cabeza de lista de los grupos más conservadores muestra el intento de paliar el déficit de legitimidad democrática con el que cuentan. Por otra parte, la presencia de Rafsanyani en la lista de los conservadores impidió que su grupo "Servidores de la Reconstrucción" consensuara una lista común con el Frente 2 de Jordad, a pesar de que ambos presentaran varios candidatos comunes, entre ellos Hadí Hamenei -hermano del Guía, pero muy cercano a la línea de Jatami-, o Reza Nuri -hermano del ministro encarcelado-, o la periodista Yamila Kadivar -esposa del ministro de Cultura, Ataollah Mohajerani, destacado "Servidor de la Reconstrucción", y hermana de Moshen Kadivar.

La ausencia de Nuri, enjuiciado y dejado unos días en libertad provisional, ha impedido a los reformistas ponerse de acuerdo en un nombre para encabezar el cartel electoral, lo que ha motivado que varios de estos grupos hayan confeccionado listas ligeramente diferentes, ya que el sistema electoral irani permite que un candidato aparezca en varias listas.

Se repetía así la actuación llevada a cabo contra Gholamhusein Karsbachi, alcalde de Teherán, en vísperas de las elecciones presidenciales de 1997.

Otra importante medida coactiva, siempre en el filo de la represión, ha sido la utilización de la ley de prensa. Una ley de prensa concebida durante los años de guerra con Irak, llena de carencias y donde hay una ausencia total de claridad sobre qué constituye un delito de prensa, lo que permite al sector inmovilista cerrar periódicos y llevar a sus editores ante los tribunales. Esta ha sido la estrategia utilizada contra Nuri, como contra otros muchos.

El sector conservador ganó una primera batalla al lograr presentar en el Parlamento, antes que los reformistas, una enmienda a esta ley vigente, para endurecerla aún más y apoyar su utilización de la vía judicial. En julio de 1999, en pleno fragor de la contienda por la libertad de prensa, consiguieron que se retrasara su votación mientras seguian buscando apoyos para que su propuesta prosperara.

Las represivas tienen fundamentalmente un campo de actuación, el jurídico. Y dos ámbitos importantes en el proceso democrático en marcha: represalias contra la prensa, principal instrumento de denuncia de la corrupción, la arbitrariedad y la falta de respeto a la norma, por parte de los reformistas; y el enjuiciamiento y encarcelamiento de políticos afines al campo reformista.

El Guia Supremo nombra directamente al jefe del poder judicial, quien puede poner dificultades al poder ejecutivo, incluso en los asuntos que son de su competencia, como la supresión de diarios aperturistas, cuando esta función corresponde al Ministerio de Cultura y Orientación Islámica . El artículo 36 del capítulo 3 de la Constitución dice que "una sentencia no puede pasar más que por un tribunal competente y de acuerdo con la ley", lo que quiere decir que nadie puede invocar únicamente la sharía y que la ley pertenece al dominio del estado. Son múltiples los casos de represión judicial sobre la libertad de expresión.

En la misma línea debe situarse la reclusión domiciliaria del ayatolá Husein Alí Montazeri, permanente crítico del poder del Guía o el enjuiciamiento de políticos progresis- 
ta, como el llevado a cabo contra Karbastchi acusado formalmente de financiar la campaña electoral presidencial con fondos públicos, aunque no puede descartarse la existencia de otras razones, como su intención de establecer un sistema municipal impositivo, o políticas, pues la condena por corrupción de Karbastchi,cuando la prevaricación es moneda corriente dentro del aparato del estado, tuvo más que ver con un ataque al ejecutivo de Jatamí que con una voluntad de saneamiento del sistema, dado que Gholamhusein Karbastchi había contribuido a privar a los conservadores de su mayoría absoluta en el Parlamento en las elecciones de 1996,y se había vinculado a la candidatura de Jatami en las presidenciales.

Los intelectuales, por su capacidad crítica, son objeto de continuo acoso, como en todo sistema cerrado.

En vísperas de las elecciones municipales de 1999, se detuvo por el Tribunal especial del Clero, al hoyattoleslam Moshen Kadivar que representa la tendencia intelectual dentro del clero crítica con el velayat y que justifica la democracia parlamentaria .Kadivar fue acusado de minar el sistema de la República islámica, insultar al imán, instigar a la opinión pública y apoyar a ayatolá disidente Montazeri. La decisión de su detención fue muy criticada por el Ministro de Cultura y Orientación Islámica.

Por último, la represión mediante la violencia física utilizando a los Ansar é-Hezbolá -militantes populistas: Compañeros del Partido de Dios- que han multiplicado sus agresiones contra las expresiones culturales de la apertura.

\section{ESTRATEGIA DE LOS REFORMISTAS}

La estrategia reformista genérica, no sólo coyuntural ante las elecciones, tiene dos campos fundamentales: los tribunales y la prensa.

Los tribunales como respuesta firme al principal campo de actuación de los conservadores -el campo jurídico- a la vez que como institución que necesita democratizarse ya que actualmente el juez es a la vez fiscal. La prensa, como instrumento de movilización y persuasión social con el proyecto reformista, y sobre todo como canal de información de las irregularidades cometidas por los círculos que detentan el poder. Ámbitos que constituyen el centro esencial de la politica interior de Jatami, y que son a la vez respuesta a las estrategias inmovilistas.

El éxito más decisivo de Jatami ha sido sacar el debate político de las altas esferas del poder y llevarlo hasta la sociedad, consiguiendo así que ésta esté informada, y animándola a participar, consiguiendo con esta táctica, que se frenen la corrupción, los incumplimientos de la ley, etc. Todo ello le ha servido no sólo para denunciar los excesos políticos existentes sino, en una labor pedagógica muy sutil y bien trazada, explicar a los ciudadanos principios teóricos como "estado de derecho"; o mediante la posibilidad de presentar la variedad de opiniones existentes en la sociedad irani, lo que significa "democracia" o "multipartidismo", o su famoso "diálogo entre las culturas".

Uno de sus objetivos nucleares estrechamente ligado con la noción de democracia y estado de derecho es acabar con los tribunales excepcionales existentes -Tribunal especial del clero, Tribunal especial de la prensa, etc.- establecidos al margen de la Constitución . 
Mediante un mensaje aparentemente simple como "la defensa de la libertad de expresión", se producen sus incursiones en los más diversos campos, incluso en el jurídico. De forma que ante las medidas coactivas y represivas dirigidas contra políticos o intelectuales como Montazeri, Kadivar, Abdullah Nuri, Kartsbachi, Soroush, y tantos otros políticos y periodistas, incluido Reza Jatami, hermano del presidente, su opción es la defensa de los acusados. A nadie se le oculta que más allá de la defensa de la libertad de expresión, lo que se está poniendo en tela de juicio en el debate y en la confrontación de estrategias es el principio de disociación y de diferenciación entre lo religioso y lo político, aunque se presente bajo formulaciones ideológicas diversas. De hecho el ayatolá Montazeri, aún bajo arresto domiciliario, en vísperas de las elecciones declaraba que "estimaba que los jefes religiosos no debían intervenir en asuntos económicos e internacionales del país y que el Guía, según la Constitución debía ser elegido por el pueblo y no por un grupo de teólogos ${ }^{18}$.

Ello se ve reforzado por el apoyo que los universitarios han dado a la discusión actual sobre el "Velayat é-faqih", abriendo reuniones públicas en la propia Universidad de Teherán.

La batalla de la prensa ha resultado la batalla más dura. Si desde la llegada de Jatamí a la presidencia ha habido una explosión de la prensa que alcanzó unas cotas de libertad desconocidas, incluso para el mundo occidental, y se ha abierto el camino para una prensa independiente y comprometida con el proyecto reformista, los periodistas han sido continuamente perseguidos, encarcelados, enjuiciados hasta que la represión ha llegado a ser directa, y muchos periodistas han perdido su licencia para trabajar. En los meses previos a las elecciones, la mayoria de los periódicos y revistas que les apoyaban han sido cerrados .

El apoyo de la prensa ha sido fundamental para los reformistas. La libertad de crítica, iniciada por Jatamí durante su etapa como Ministro de Cultura y Orientación islámica, ha permitido que algunos periodistas hayan aprovechado la coyuntura electoral para desenmascarar ${ }^{19}$ la figura del poderoso Rafsanyani, acusado de prevaricación, corrupción, nepotismo, y con posibles implicaciones en los asesinatos de intelectuales llevados a cabo a finales de 1998.

No es posible afirmar que la publicación de estas acusaciones sobre Rafsanyani hayan sido la exclusiva razón que explique su derrota electoral, posiblemente el desafecto de los iranies a cuanto no significa cambio, y el aumento de una mayor exigencia ante el comportamiento de los políticos, han tenido decisiva importancia. El resultado no se limita a la derrota electoral de Rafsanyani, y a su humillación, ni al manifiesto descrédito de la coalición conservadora, sino que ha creado un precedente importante que los restantes políticos tendrán que tener en cuenta.

Y la contraofensiva del stablishment no se ha hecho esperar, el 6 de agosto, el Guía supremo impidió el debate parlamentario previsto para reformar la ley de prensa, una de las promesas centrales de la campaña de los reformistas. El orden del día incluida el debate y

18 Entrevista publicada por "The Guardian" el 14-1-2000. 19 Dossier de prensa elaborado a lo largo de 1997 - 2000: sobre todo The Indepent, febrero 2000; El Pais, 9 marzo
2000 . 
la votación de enmiendas a la dracoiana ley de prensa aprobada por el Parlamento saliente. Como su aprobación estaba garantizada, la intervención de Jamenei que tiene la última palabra en los asuntos de Estado, obligó a suspender el debate y provocó numerosas protestas y peleas en el hemiciclo ${ }^{20}$.

Ante las medidas preventivas previas a las elecciones legislativas, también los jovenes del grupo "Oficina para la Consolidación de la Unidad" han ideado fórmulas para esquivarlas. Quizás las más ingeniosas han estado relacionadas con la prohibición de la campaña electoral mediante grandes carteles o vallas publicitarias, y sus alternativas han sido variadas; además de utilizar los permitidos folletos manuales, han elaborado miles de listas cerradas que comenzaron a funcionar por todo el pais, intentando conseguir que millones de personas se pusieran de acuerdo sobre la lista que querian votar -aunque podian hacerse variaciones por parte del votante- sin tener que difundir públicamente esta iniciativa. Es decir la elaboración de "listas-sugerencia" formadas no necesariamente por miembros de un único grupo sino también pueden ser simpatizantes o independientes. A la vez que nueve periódicos reformistas la incluían en su publicación.

Por otra parte, los activistas del Frente de Participación han montado centros de campaña en casi todas las provincias donde jóvenes militantes y voluntarios hacen llamadas en busca de apoyo, recogen donativos, previa entrega del correspondiente boleto de 5000 ó 10.000 riales ( 100 y 200 ptas) o se organizan por turnos para el reparto de propaganda, financiándose así la campaña.

Por su parte, la campaña electoral se ha caracterizado por la modernidad de sus contenidos, dejando en último lugar los mensajes religiosos o moralistas y Jatami se ha centrado en los temas que afectan al conjunto de la población: libertades, seguridad pública, desarrollo social o necesidad de reformar un sistema político con mayor estabilidad para el futuro. Además de la utilización de símbolos y referencias pre-islámicos y eminentemente persas, como el uso de la imagen de Ferdushi, padre de la literatura persa y emblemático símbolo de la independencia nacional. No es la primera vez que esta simbologia es utilizada ${ }^{21}$, Jatami, tras los incidentes de julio 1999, recibía un baño de multitudes en Hamadán a $300 \mathrm{Kms}$ al suroeste de Teherán, una ciudad con profundas raices persas.

Todo ello sin abandonar la inclusión de la música o la tecnología más actual, desde el uso del "pop" iraní que ha acompañado a los actos del Frente de Participación, pasando por el alquiler de establecimientos de lujo para sus actos, como el restaurante Top, en Teherán, lugar muy cotizado ya en los tiempos dcl Sha, hasta la creación de una página web del Frente de Participación a la que se podían realizar consultas .

Todo ello no ha impedido hablar de asuntos internacionales, y algunos líderes como Reza Jatamí, han pedido que se respete el proceso de paz iniciado por Arafat; y Jatami, refiriéndose a las relaciones con Estados Unidos dijo que si los jóvenes de 1979 estuvieron legi-

20 El Pais, 6 agosto 2000.

21 Ya con Rafsanyani se dio un recentraje de Irán en torno a las viejas provincias farsis, en ROY, O.: Géopolitique de l'Asie centrale" en Cahiers du monde russe et sovietique. XXXII, n ${ }^{\circ}$, enero-marzo 1991, p, 1.136. 
timados para ocupar la embajada de Estados Unidos, las nuevas generaciones lo están para tomar iniciativas encaminadas a acercar los dos sistemas políticos enfrentados desde hace veinte años ${ }^{22}$.

Los universitarios volvieron a hacer gala de imaginación organizando un "contramitín" en el que el protagonismo giró en torno a tres aspirantes a candidatos cuya participación en las elecciones había sido rechazada por el Consejo de Vigilancia, aprovechando la oportunidad para pedir la libertad de Abdullah Nuri y Moshen Kadivar. Para después de una breve intervención de Reza Jatami, los organizadores dieron la palabra a los jóvenes para que plantearan preguntas a los candidatos fallidos, cntre ellos a Abbas Abdi, director del periódico "Salam" cuyo cierre motivó las protestas estudiantiles de julio de 1999 .

Si he resaltado algunos rasgos de la estrategia electoral, que desde una lectura superficial no mostrarian más que lo anecdótico de una campaña más coloreada que la de los adversarios, una lectura con menos" ruido" informativo permite descubrir que la misma forma de organizar la campaña tiene otra función más importante, la de incidir en el ámbito de las costumbres, para normalizarle.

Una característica importante en las campañas electorales e intervenciones públicas del presidente Jatami ha sido que nunca ha quebrantado la Constitución, dando ejemplo de lo que significa la supremacía de la ley, y sometiéndose a ella, sin abandonar sus principales postulados: la defensa de las libertades, la compatibilidad del islam y la libertad, y del islam y la cultura. A los veinte días de las manifestaciones estudiantes de julio de 1999, Jatami dijo en su intervención pública: “ Ellos -en referencia a los inmovilistas-intentan decir que la religión y la libertad no pueden convivir y aseguran que las universidades representan un peligro para el islam y la revolución. Pretenden decir que la seguridad y la libertad no pueden coexistir, y que para mantener la libertad, las libertades deben ser cercenarse. Pero la nación no será confundida(...)." ${ }^{\text {"23 }} \mathrm{O}$ " (..) se trata de establecer el Estado de derecho combinado con los principios islámicos, de defender el Estado de derecho en el marco de un Estado islámico. No se trata de superar la revolución islámica de 1979 , sino de continuar el camino iniciado por ella"24.

El distanciamiento de los" nuevos islamistas" respecto del islam oficial ha provocado un enfrentamiento entre ambos que se trasluce en una mutua animadversión.

Lo llamativo es que los métodos utilizados por los más intransigentes en el poder no ha generado una oposición política violenta. La oposicion islamista ha sabido responder con métodos políticos a lo que es un problema de tal índole ${ }^{25}$. De ahi, en gran parte, debe su éxito electoral el islamismo reformista.

22 El Mundo 17 -II- 2000; El Pais, 13 y 15 -II- 2000.

23 The Indepent, 29 julio 1999.

24 El Pais, 30 abril 1997.

25 All Asthrî, portavoz de los estudiantes iranies en julio de 1999, declaraba que Jatami les habla enseñado a no responder con violencia a la violencia, sino de forma lógica y con respaldo popular. 
En definitiva, el proceso democrático está poniendo en evidencia que el sistema político irani funciona en un equilibrio inestable donde el consenso predomina en la toma de decisiones importantes, y en el plano religioso que el neofundamentalismo conservador, cuya mayor preocupación es el fiqh y las costumbres, no ofrece a una sociedad que ha cambiado y se ha rejuvenecido, ninguna alternativa para su inserción en el mundo actual. 